\title{
Spatio-temporal effects in visual gap detection ${ }^{1,2}$
}

\section{WILLIAM R. UTTAL ${ }^{3}$ AND RAMELLE HIERONYMUS}

UNIVERSITY OF MICHIGAN

The ability of the visual system to process temporal information is studied with regard to the interactions between temporal and spatial stimulus dimensions. A comparison of the results of two experiments indicate that the eye is able to discriminate temporal gaps in a train of visual flashes better when the flashes are spatially superposed then when they are dispersed. Thus, the inertial and integrating properties of the visual receptor seem, at first glance, to hinder the discrimination less than does spatial dispersion. However, an analysis of the results, using Levinson's low-pass filter model, suggests that the increased discrimination is due to the recoding of the temporal stimulus information along a brightness dimension, and thus the true temporal properties of the visual system are probably better described by the dispersed rather than the overlapped condition. Other experiments are also reported that deal with related phenomena germane to the ability of human $S$ s to deal with the temporal information pattern of visual stimuli.

Recently we have been studying a visual temporal discrimination task in which dotted alphabetic characters are mixed with randomly placed dots on the face of an oscilloscope. Depending upon the temporal relations between the dots of the visual noise and the dots of the character, a variable degree of confusion was obtained, which is reflected in the recognizability scores of the character set.

In one experiment (Uttal, 1969b), the character was prèsented within a temporal hole or gap between a burst of leading noise and a burst of trailing noise. The recognizability of the character under these conditions was relatively high, indicating that a break or gap, appearing in the stream of noise dots, allowed the character to show through. A question thus arises: How small a gap or break can be detected in such a visual stimulus?

The problem, thus phrased, is one that has concerned psychologists for many years. Temporal acuity in vision has been studied in many different paradigms. Basler (1911) studied the two-flash situation with identical flashes and found that, at a 35-msec interval, the gap between two $35-\mathrm{msec}$ flashes could not be detected More recently, Lindsley and Lansing
(1956) showed the minimum detectable interval in a two-flash situation to be about $70 \mathrm{msec}$. As Brown points out (see Basler), the minimum interval that can be detected is substantially reduced by repeating the visual stimulus; this, of course, is the classical flicker fusion experiment. Under these conditions, additional information is presumably available to the nervous system that allows a finer temporal discrimination to be made and smaller intervals to be detected than would have been if only a pair of flashes had been presented. Current estimates of critical flicker fusion frequency under optimal conditions usually suggest that $60-70 \mathrm{~Hz}$ is the upper limit of visual temporal discrimination (see Landis, 1954). Under some conditions (Alpern, personal communication, 1969), indirect effects of $100-$ to $110-\mathrm{Hz}$ flickering lights have been detected However, a typical characteristic of most of the flicker experiments that have thus far been carried out is the use of a single source stimulus, activated in some temporal pattern. Similarly, the durations of all the dark intervals in a conventional flicker stimulus are equal, and the ability to detect a flicker is a statistical phenomenon measured over many in tervals rather than the estimate of a single gap.

Like many of the experiments in backward masking (see Uttal, 1969a), this type of stimulation may also confound what may be two separable phenomena: peripheral (retinal) interactions and purely temporai discriminations that might still be obtained if the inertial effects of the periphery could be bypassed.

In the present study," we have mainly used the same sort of spatial pattern of signals appearing in several of the early studies-dynamic visual noise (DVN). DVN appears as a dancing pattern of tiny dots plotted in random spatial locations at regular temporal intervals. Because each dot in the DVN is plotted with a rather low probability of occurrence at any given point, the probability is very low that any spot on the CRT screen (and thus any retinal location) will be activated twice in succession. In this case, the spatial discreteness of the separated dots introduces a set of additional factors that could also elevate the threshold for gap detection. It is also possible, however, to plot all of the points in a single locus. Thus, we have an added control that can help to distinguish between spatial interaction effects and purely temporal resolutions.

The results of several experiments are reported in this paper. The first experiment tests the ability of the $S$ to discriminate a discontinuity or a gap in a burst of dynamic visual noise.

The second experiment was carried out to compare the results of the first experiment with the more usual gap detection or flicker paradigm in which the output of a single stimulus source is modulated. This study is directly comparable to the gap test first used by us in an earlier study (Uttal \& Krissoff, 1966) of somatosensory temporal discrimination and later developed by Pollack (e.g., 1967) in an extensive set of studies of auditory gap discrimination. In this experiment, the dots were all plotted at exactly the same rates as in the first experiment, but all were superimposed upon each other in one central location. Since the light output of the CRT used in the present study was of extremely short persistence, there was still a nearly identical temporal pattern maintained even though the spatial dispersion was completely eliminated. The results of both Experiments 1 and 2 were notable in their suggestion that the visual system is able to maintain some information about stimulus frequencies up to about $1,000 \mathrm{~Hz}$, an effect previously noted by Levinson (1968) with what he termed "half sinusoids"; however, this information is probably maintained in terms of a brightness discrimination rather than a pure temporal discrimination.

Next, because the number of light. flashes had been so important in determining the threshold for the gap in the older critical flicker fusion experiments, we conducted a third experiment in which the number of randomly positioned light flashes before and after the gap was varied. This was done to determine if there were any systematic changes in the gap threshold as a function of this variable in our present experimental configuration. We found that the number of dots was an effective variable in specifying the size of the smallest detectable gap.

The final experiment repeated the design of the first, but used another cathode ray tube with a medium persistence light trace. Differences in the results could then be 
attributed to the differences in the phosphorescent decay rates of the two cathode ray tubes (CRTs) and also provide an additional suggestion about the persistence of the visual trace itself. All these studies examine the problem of the time constants of the visual system.

\section{METHOD}

Subjects

Four female Ss served throughout this experiment. Each $S$ worked for $1 \mathrm{~h}$ a day. Little pretraining appeared to be necessary. After the first 2 days of familiarization with the experimental procedure, no further overall diminishment in the values obtained for the threshold for identical conditions could be observed.

\section{Apparatus}

The $S$ was seated in a darkened and acoustically insulated booth with the bridge of the nose positioned $14 \mathrm{in}$. in front of the face of a Tektronix 561A oscilloscope. A special CRT was used in this instrument. The face of this CRT was coated with a nonstandard P-15 short-persistence phosphor that decays in brightness to $0.1 \%$ of the original brightness in 50 microsec. Thus, plotted points presumably dropped to subthreshold brightness levels in about this brief period. A typical experiment used as a display a patch of dynamic visual noise, approximately $2 \frac{1 / 4}{4} \times 1 \frac{1 / 2}{\text { in. }}(9 \times 6 \mathrm{deg})$. Because of the persistence of the visual image, there appeared to be a large number of randomly positioned dots dancing on the screen of the CRT at any given instant.

A small digital computer (a PDP-5 manufactured by the Digital Equipment Corporation) was used to control the presentation and timing of the visual patterns. The electron beam of the CRT was controlled in position by the output of a two-channel digital-to-analog converter. This device digitized randomly selected $x$ and $y$ coordinates to select the points at which a dot would be plotted. A third signal (the $z$ axis) intensified the beam for a period of 10 microsec, after which the natural decay of the P.15 phosphor determined when the visual stimulus would be discontinued. Like most phosphors, this one decayed in brightness exponentially following the cessation of the intensification signal. Some additional considerations concerning this sort of visual stimulus generator were detailed in one of our earlier papers (Uttal, 1969a).

\section{Procedure}

All aspects of the experiment were controlled by the computer. Stimulus generation occurred after a constant 1.5-sec delay following the S's response to the previous stimulus. The specific psychophysical procedure utilized was a two-alternative forced-choice design in which the $S$ was presented with two sequential stimulus patterns (separated by an interval of about $1.5 \mathrm{sec}$ ) and required to say which contained a temporal discontinuity (a gap or a break in the sequence of dots). To respond, the $S$ held two pushbuttons in his hands, each of which was coded to represent either the first or the second stimulus burst. A contingent experimental design was used such that a correct response (the choice of the stimulus with the gap) led to a reduction in the size of the gap in the next presentation by $3 \mathrm{msec}$. On the other hand, if the $S$ was mistaken in his choice, the size of the gap would be increased by $9 \mathrm{msec}$ for the next trial. This imbalance, while adding a slight upward bias to our estimates of the threshold, does tend to prevent any completely inattentive $S$ from giving a series of random (or entirely one-sided) responses, which could lead to a false, though apparently stable, threshold measurement. Each $\mathrm{S}$ spent $1 \mathrm{~h}$ each day in the experimental cubicle going through 8-12 blocks of trials. In each block, the value of the independent variable was held constant but the order of the blocks was randomized. Each block consisted of 50 trials, but only the last 40 were used in computing the average value of the threshold for each block, the first 10 being used by the $S$ to "hunt" the general region of the threshold for these particular conditions. The average values for each condition were pooled across all $\mathrm{Ss}$ and all sessions by the computation of a grand mean. Since dark adaptation was a significant variable in this experiment, a pair of dummy blocks preceded the actual data-collection blocks.

At the end of each block, the $S$ was instructed to rest for about $1 \mathrm{~min}$. The indication to her that a block had been completed was the prolonged typing of a summary statement on the console typewriter. This teletypewriter also typed out a complete protocol of the experiment, listing the current gap size and the S's response on each trial. In this way, the $E$ could monitor the course of the threshold tracking process at his leisure.

In Experiment 1, each stimulus presentation consisted of two sequential bursts of DVN with the dots dispersed in random positions across the same viewing region on the face of the CRT. The independent variable in this experiment was the interdot interval between sequential dots of this spatially dispersed visual pattern. One of the bursts (chosen at random by a one-bit random number generator) had the gap in it, the detection of which was the S's assigned task. The duration of each burst of DVN dots was $1 \mathrm{sec}$ plus, in the case of the burst with the gap in it, the duration of the gap. The Ss participated in four 1-h-long sessions on successive days, using interdot interval values of $1,3,5,7,10,13,15$, and $20 \mathrm{msec}$. The first experiment was repeated on 4 days and all data were pooled.

In Experiment 2, the $\mathrm{x}$ and $\mathrm{y}$ coordinate inputs of the oscilloscope were set to the "ground" position to fix the position of all dots at the center of the oscilloscope. All the sequential dots were thus plotted on top of each other, but in other respects all the conditions of Experiment 1 were replicated. The second experiment was also repeated on 4 days and all data were again pooled.

In the third experiment, the independent variable was the number of dots in the bursts rather than the interdot interval. In this experiment, the numbers of dots used in successive blocks were 10 , $20,30,40,50,60,70$, and 80 . In this case, since the interdot interval was kept constant at $7 \mathrm{msec}$, the duration of the two bursts varied over a wide range. This experiment was also repeated on 4 days and all data, as usual, were pooled.

In Experiment 4, once again all the conditions of Experiment 1 were replicated with the exception that in this case a medium-persistence P-31 phosphor CRT was used.

\section{RESULTS}

Figure 1 presents the results of Experiments 1,2, and 4.

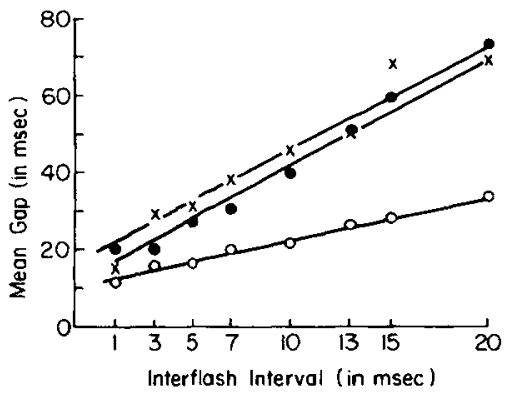

Fig. 1. The results of three of the experiments are plotted in this figure. The filled circles depict the results of Experiment 1 in which the $S$ was required to detect a gap in dispersed random visual noise. The empty circles represent the results of Experiment 2 in which all the dots in the pulse train were plotted on top of each other in a central location. The $x s$ plot the results of Experiment 4, in which the ultrashort-persistence (P-15) CRT was replaced with a medium-short-persistence one (P-31), and the paradigm of Experiment 1 then repeated. 


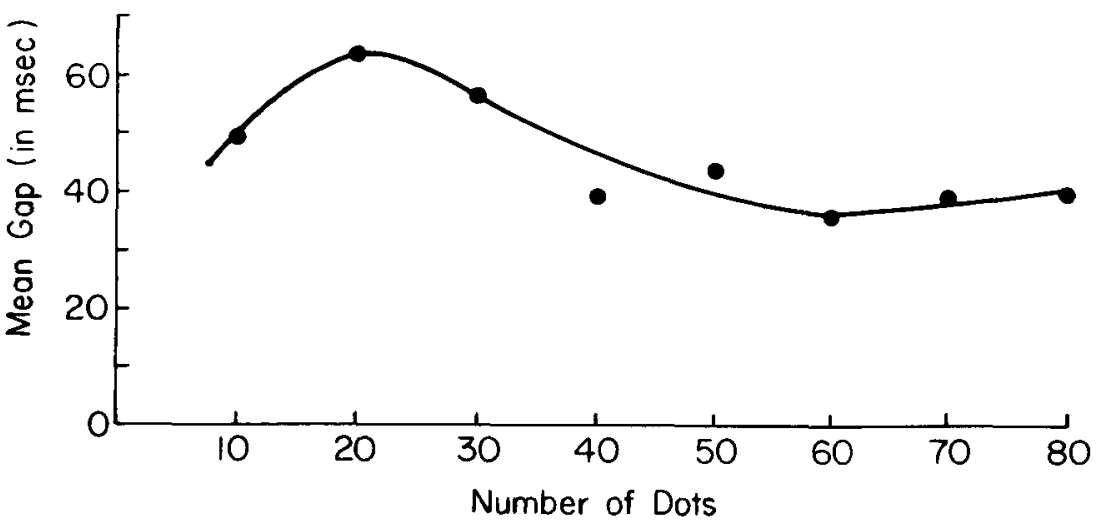

For all these experiments, the abscissa represents the interdot interval between the dots in each of the two bursts of each trial, while the average value of the just-detectable gap is the dependent variable, indicated on the ordinate. As the interdot interval increases, the size of the gap monotonically increases for all experiments. Nevertheless, the values for the spatially distributed dots are noticeably higher than those for the single fluctuating point. Of considerable interest is the fact that even at interdot interval values that are considerably less than the shortest measured gap, the interdot interval is still conveying behaviorally usable information. Thus, for example, the difference between a 1- and 3-msec interdot interval in the single dot paradigm of Experiment 2 is significant, even though both $1,000-\mathrm{Hz}$ and $333-\mathrm{Hz}$ repetition rates are considerably above all previous estimates of critical flicker fusion rates. A similar condition occurs if one compares the 3. and 5-msec conditions of Experiment 1. Here there is what appears to be a significant difference in the average gap sizes, in spite of the fact that a comparison is being made between stimulus repetition rates of $333 \mathrm{~Hz}$ and $142 \mathrm{~Hz}$, respectively. Though the $S$ is unable to detect these brief interdot intervals as measured with the techniques of the present experiment, he is capable of discriminating, on some behavioral basis, that the dot repetition rate is not the same in each case. Since the durations of the bursts are controlled and always equal to $1 \mathrm{sec}$, it is unlikely that the $S$ is discriminating on the basis of any duration criterion. We shall discuss below the reasons for believing that this is, in fact, a discrimination based on a recoding of the interval information into brightness information under the conditions of Experiment 2.

It is also interesting to note that, in the case of Experiment 2, the results are closely analogous to the earlier work done in our laboratory on gap detection in the somatosensory system (Uttal \& Krissoff,
1966). The single point of light in the present paradigm can properly be compared with the one-dimensional train of electrical pulse stimuli used in that earlier study. Picking two points that are directly comparable (interpulse in the somatosensory experiment or interdot intervals in the present experiment equal to $10 \mathrm{msec}$ ), which were both obtained using the equivalent two-alternative forced-choice design, we find the scores nearly identical: 20 and $22 \mathrm{msec}$, respectively. Thus, the two situations suggest temporal processing capability that is of the same sensitivity in the two modalities, a somewhat surprising result considering traditional discussions of the integrating functions of the retina.

Figure 2 displays the results of the third experiment in which the number of dots on either side of the gap was varied. The curve shows a gradual increase in the S's ability to detect the gap as the number of dots is increased to about 50 or 60 . However, the single value of an $N$ of 10 dots on either side of the gap is depressed from this general curve. This result has been replicated several times, using different sequences of block conditions, but no explanation can be provided for this unusual result at this time.

The replication of Experiment 1 is also plotted in Fig. 1, using the longer-persistence P-31 phosphor. The effect of this longer persistence can be seen to be relatively slight; little difference is observed when a comparison is made with the results of Experiment 1.

\section{DISCUSSION}

Several issues of interest arise from the data we have obtained in the present series of experiments. The most basic question of all concerns the basis upon which the gap is "seen." The subjective experience is of considerable significance here and is generally reported for all the conditions we used to be a "wink" in an otherwise continuous visual experience. For a brief moment, the light appears to cease and
Fig. 2. The results of Experiment 3 are plotted in this figure, showing the $U$-shaped function produced by varying the number of duts on either side of the gap. No explanation can be provided for this somewhat surprising result.

there is a momentary dark temporal hole. In the case of the distributed dots of Experiment 1, this wink must necessarily be contrasted with the almost continuous winking of the various dots as they appear at one position or another across the face of the CRT. The results of our Experiment 1 indicate that the discrimination of the "wink" from the hopping of the dots is more of an interference than an inertial effect created by the continuous stimulation of a single retinal locus in the paradigm used in Experiment 2.

The nature of the subjective reports suggests that the ability to perform the temporal gap-detection task we are studying is, in fact, based upon a recoding of the temporal information into an intensity dimension, a possibility that helps us to make sense of what appears to be an incre tibly high temporal discrimination ability on the part of our Ss. Levinson (1968) has proposed an interesting model, using the notion of cascaded low-pass filters or simple integrators to explain some aspects of the discrimination task in Experiment 2. One important aspect that his model explains is how a series of cascaded low-pass filters with an arbitrary but reasonably chosen time constant can maintain coded information about the input frequency without itself maintaining specific frequency information. Figure 3 shows a diagram of a simple circuit model based on Levinson's description. Also pictured on this figure are the input and outputs of the system for several different interflash intervals $(1,1.6,2,4$, and $8 \mathrm{msec}$ ) as well as a plot of the amplitude against both interflash interval and frequency. The RC circuit in this primitive model is essentially acting as a simple analog computer with the light pulses analogized by brief electrical pulses of equivalent duration. This cascaded system of integrators represents a functional model of the visual system; no thought should be given to ascribing specific neurophysiological correlates to each of the integrating stages. Nevertheless, it can be seen that the amplitude of the output signal, corresponding to the perceived brightness, is a linear function of the frequency of the stimulus light flashes. Since no psychophysical data have been collected for amplitude estimates with this particular kind of stimulus, it is not 

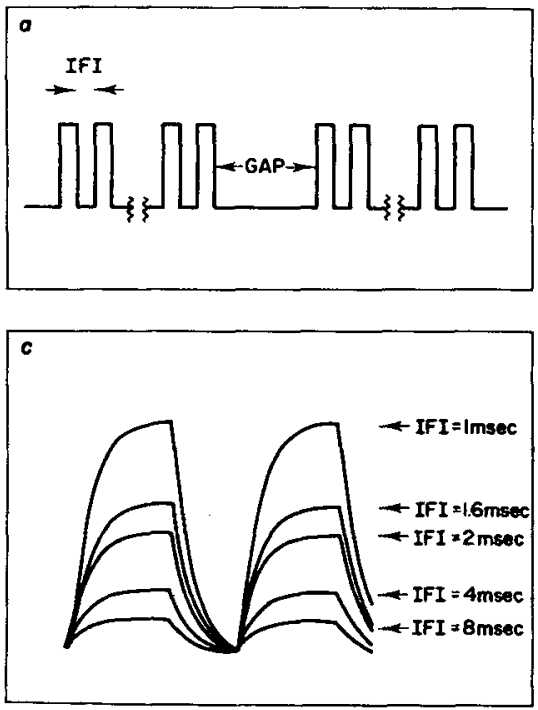

possible for us to relate brightness and interflash interval directly. However, the reciprocity relationship (Bloch's law) and the Talbot-Plateau law would suggest that the brightness should be linear with regard to the frequency, just as is the model.

Now let us consider what might possibly be the critical criterion for the detection of the gap, assuming that it is indeed a brightness discrimination task. The rise and the decay of the simulated brightness in this model can be seen to have essentially exponential characteristics. Thus, if we were to assume that the dark "wink" was observable when a constant amount of darkening $(\Delta B)$ had occurred, one might expect that there should be a similar increase in the duration of the threshold gap as the initial brightness $\left(\mathrm{B}_{\mathrm{o}}\right)$ decreases. This would be so because, as $B_{o}$ is lessened, it simply would require an exponentially increasing time to decay the brightness equal absolute amounts, in accord with the exponential decay curve. Our data, on the other hand, display a linear increase in gap size, thus contradicting the notion that a constant amount of darkening is the key cue for the detection of the gap.

Fig. 4. This diagramatic sketch presents the gap size as a function of IFI assuming certain relationships between the change in brightness $(\Delta B)$ and the initial brightness level, $B_{O}$. The uppermost curve represents the exponential increase in gap size that would be expected assuming that $\Delta B$ was a constant. The bottommost horizontal line shows the constant gap size that would have been expected assuming that $\Delta B / B_{0}$ was a constant. The actual results of Experiment 2 are plotted along with one plausible form of the relation between $\Delta B$ and $B_{O}$ as derived in the text.
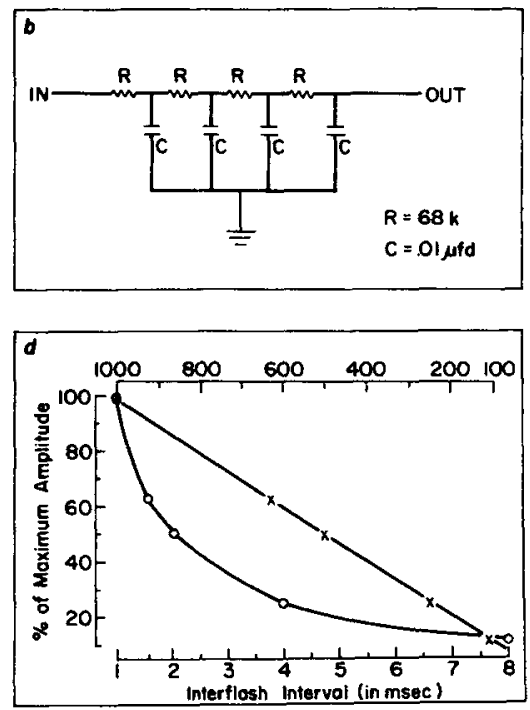

An alternative possible criterion is that a constant contrast ratio $\left(\Delta B / B_{0}\right)$ is the critical cue. In other words, according to this hypothesis, the critical information that a gap had occurred would be a constant proportion of brightness decrease rather than an absolute magnitude of dimming. However, exponential curves operating in a system of this sort, in which the time constant presumably remains constant, all decay to an identical percentage of the initial values in identical periods of time, independent of the amplitude of the initial brightness. Therefore, the $50 \%$ brightness level, for example, occurs for all values at exactly the same time, and there would be no basis for any difference in the minimum detectable gap as a function of $\mathrm{B}_{\mathrm{o}}$. Furthermore, Graham and Kemp (1938), among others, have shown that $\Delta \mathrm{B} / \mathrm{B}$ is not constant over $B$ in more conventional brightness discrimination tasks, and this
Fig. 3. The drawings in this figure present the details of the analog model of the visual system that we have utilized (following Levinson, 1968) to explain the results of Experiment 2. Drawing a shows the train of simulated light flashes stimulating the low-pass filter depicted in Drawing b, which models the temporal properties of the visual system. Drawing c shows the output of the simulated visual system and, although drawn to completely different time scales, shows how the IFI of Drawing a can affect the amplitude of the output of the system. The output curves of Drawing c are traced from a Polaroid photograph of an oscillographic plot of the output of the model. The vertical and horizontal scale in Drawings $a$ and $c$ are both arbitrary and not to the same scale. Drawing d shows the relations between the simulated brightness and both IFI and frequency and demonstrates the prediction that the simulated brightness, $B_{0}$, representative of the predecay amplitudes is linear with regard to frequency rather than IFI.

relation should not be expected to hold in any case.

Considering the data of Experiment 1, we note that the entire range of threshold gap sizes is relatively narrow. The gaps range from $12 \mathrm{msec}$ to $34 \mathrm{msec}$ (a factor of about 3), even though the interflash interval varies over a range of 20 to 1 and the frequency over a range of 8 to 1 . This suggests that the actual curve relating $\Delta B$ to $B_{0}$ lies somewhere between the first and second alternatives described above, i.e., between the exponential curve representing a constant absolute magnitude criterion ( $\Delta B=$ constant) and the horizontal line representing the constant ratio criterion $(\Delta B / B=$ constant $)$. Figure 4 depicts the region between these curves in which the

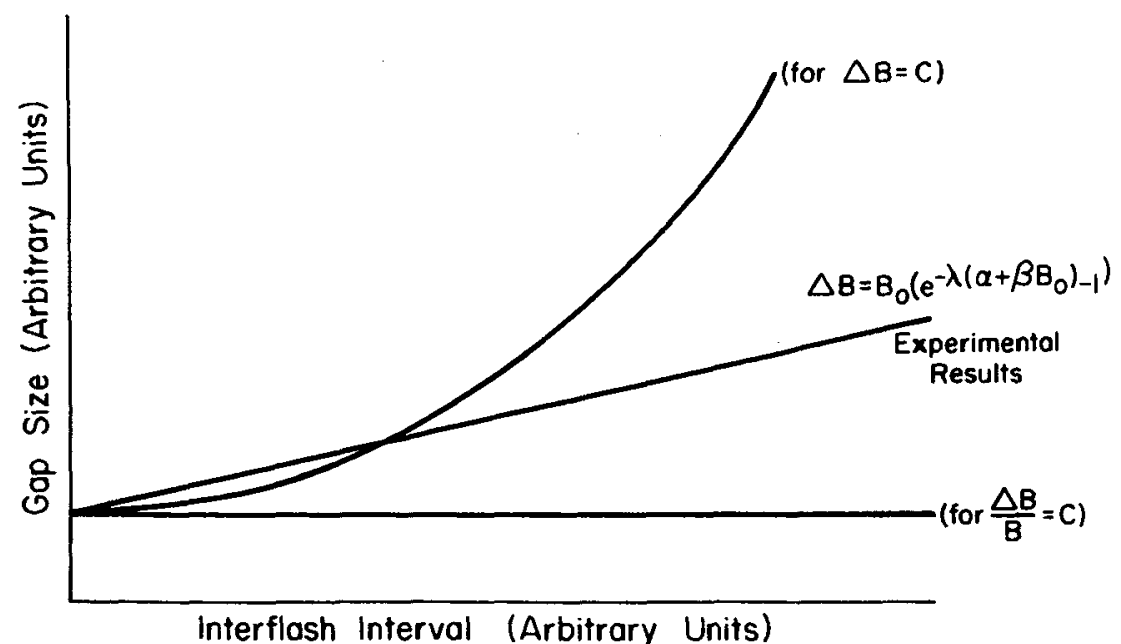


actual empirical data would be expected to lie.

It is possible to estimate the functional relationship between the required change in the subjective brightness $(\Delta B)$ and the initial brightness $\left(B_{O}\right)$ by making certain assumptions about a plausible and possible relationship between interflash interval (IFI) and $\mathrm{B}_{0}$.

The experiment itself provides the specific form of the psychophysical relation between threshold gap size and the IFI. It is a straight line and can be represented by

$$
g=a+b I F I
$$

where a, the intercept with the IFI axis, is empirically -9 and $b$, the slope of the line, is empirically 1.17. In this case, we will assume that $B_{0}$ is linearly related to IFI, a simplifying speculation that we have already suggested may in fact not be true. If it is not, it would require a simple correction in some future reformulation, but for the purposes of this semiqualitative discussion, in which we are only looking for the general nature of the criterion for the detection of the gap, it is an acceptable approximation. Equation 1a thus becomes

$$
g=\alpha+\beta B_{0}
$$

We now must consider the general nature of the decay of the brightness from the $B_{0}$ level as the gap occurs. We assume, in accord with the integrative resistor-capacitor model proposed by Levinson, that the decline is an exponential such that the brightness at any time $t\left(B_{t}\right)$ can be represented as

$$
B_{t}=B_{o} e^{-\lambda t}
$$

wherein $\lambda$ is the reciprocal of the time constant of the visual system.

Keeping in mind that our main goal is to find a relationship between the amount of change in brightness $(\Delta B)$, the difference between $B_{o}$ and $B_{g}$ (the brightness when the gap is detected), we can take the difference

$$
\Delta B=B_{g}-B_{o}=B_{o} e^{-\lambda g}-B_{o}
$$

where $g$ may be substituted for $t$ since $g$, the gap duration, is equal to the decay time $t$ at which the gap is detected.

Then dividing by $\mathrm{B}_{0}$,

$$
\frac{\Delta B}{B_{o}}=e^{-\lambda g}-1
$$

or

$$
\Delta B=B_{o}\left[e^{-\lambda\left(\alpha+\beta B_{0}\right)}-1\right]
$$

which is a plausible form of the general relation we sought specifying the size of the change in brightness required for the $S$ to see a gap as a function of the initial brightness before the gap, and assuming a linear relation between $B$ and IFI. We reiterate, however, that the relationship between $B_{0}$ and IFI is speculative and Eq. 5 might differ somewhat in a final analysis based upon some more definite assessment of this relationship.

Another advantage of this formulation is that it will also allow us to estimate the time constant $\left(T_{c}\right)$ of the visual system. Rearranging and taking the logarithm of both sides of Eq. 5 gives

$$
\ln \left(\frac{\Delta B}{B_{o}}+1\right)=-\lambda\left(\alpha+\beta B_{o}\right)
$$

or

$$
\ln \left(\frac{\Delta \mathrm{B}+\mathrm{B}_{\mathrm{o}}}{\mathrm{B}_{\mathrm{o}}}\right)=-\lambda\left(\alpha+\beta \mathrm{B}_{\mathrm{o}}\right)
$$

Now, since $\left(\Delta B+B_{o}\right)$ is equal $(\Delta B$, of course, always being negative) to the brightness of the light at the gap-detection level

$$
\ln B_{g}-\ln B_{o}=-\lambda\left(\alpha+\beta B_{o}\right)
$$

Or

$$
\lambda=\frac{\ln B_{o}-\ln B_{g}}{\left(\alpha+\beta B_{o}\right)}
$$

Perhaps more practically, it provides a perspective that may allow us to compare the time constants of the triad of chromatic receptors and thus may possibly lead to an understanding of such temporal effects as the Prevost-Fechner-Benham subjective colors (see Cohen \& Gordon, 1949 , for an excellent review).

However, one would not expect the results of Experiment 1 to be subject to these same notions of integrated and relative brightnesses. The fact that the dots are distributed over a wide region of the retina (as well as the face of the CRT) suggests that there should be no major temporal summation of brightnesses as described in the Levinson model. Thus, the results of Experiment 1 may represent a better estimate of the purely temporal discrimination properties of the visual system than those of Experiment 2. In the latter case, the temporal information of the stimulus, while discriminable, is presumably recoded into the brightness discrimination task we have described above, and it is this additional indirect use of the stimulus information pattern that results in the lower thresholds for Experiment 2.
The fact that the task of Experiment 2 was essentially a dark-bright contrast discrimination task alerted us to the probability that dark adaptation might be expected to have been a significant variable. Indeed, we generally found a substantial increase in the measured thresholds for the first two blocks of each daily session, regardless of the order of the experimental blocks. It was for this reason that these blocks were routinely discarded throughout the course of the experiment. On the other hand, this adaptation effect was not nearly as strong in Experiment 1 as in Experiment 2 as should have been expected.

The next question we should consider concerns the lack of effect of the different phosphor types of the thresholds measured in Experiment 1 and Experiment 4. The P.31 phosphor used in the slow tube is rated as decaying to $0.1 \%$ in about $10 \mathrm{msec}$. The fact that the two tubes produced virtually identical results suggests that there is another persistence in the system that is elongating the stimulus of the P-15 tube in a way that makes it ind istinguishable from the longer-persistence P-31 tube. This second level of persistence, of course, is probably the short-term storage of the visual system itself. Thus, the dynamics of the visual system slur the brief pulse of the P-15 dot over a length of time at least as long as the persistence of the P-31. The procedure of these two experiments, therefore, would be another independent way of measuring the persistence of the visual image, given a complete range of phosphor persistencies with which to work. Such a set is not currently available, but at least we can be assured that the use of the longer persistence phosphor does not significantly distort the results of this experiment.

This is of some importance, since experiments now in progress involve the use of colored phosphors to study the time constants of the trichromatic receptors, as described above, and none of the colored phosphors are available with the ultra-short persistence of the P.15 monochrome coating.

\section{REFERENCES}

BASLER, A. Über die Verschmelzung von zwei nacheinander erfolgenden Lichtreizen. Pflügers Archiv für die gesamte Physiologie, 1911, 143, 245-251. Cited by J. L. Brown in C. H. Graham (Ed.), Vision and visual perception. New York: Wiley, 1965. P. 264.

COHEN, J., \& GORDON, D. A. The Prevost-Fechner-Benham subjective colors. Psychological Bulletin, 1949, 46, 97-136.

GRAHAM, C. H., \& KEMP, E. H. Brightness discrimination as a function of the duration of the increment in intensity. Joumal of General Physiology, 1938, 21, 635-650. 
LANDIS, C. Determinants of the critical flicker-fusion threshold. Physiological Review, 1954, 34, 259-286.

LEVINSON, J. Z. Flicker fusion phenomena. Science, 1968, 160, 21-28.

LINDSLEY, D. B., \& LANSING, R. W. Flicker and two-flash fusional threshold and EEG. American Psychologist, 1956, 11, 433.

POLLACK, 1. Asynchrony: The perception of temporal gaps within periodic auditory pulse patterns. Journal of the Acoustical Society of America, 1967, 42, 1335-1340.

UTTAL, W. R. Masking of alphabetic character recognition by dynamic visual noise (DVN).

Perception \& Psychophysics, 1969a, 6, 121-128.

UTTAL, W. R. The character in the hole experiment: Interaction of forward and backward masking of alphabetic character recognition by dynamic visual noise (DVN).

Perception \& Psychophysics, 1969b, 6 $177-181$.

UTTAL, W. R., \& KRISSOFF, M. Effect of stimulus pattern on temporal acuity in the somatosensory system. Journal of Experimental Psychology, 1966, 11, 878-883.
NOTES

1. This work was supported in part by NSF Grant GB-12771.

2. We would like to acknowledge the assistance of Mr. Stuart Corwin who wrote the experimental control program. The editorial advice and research assistance of Mrs. Lynne Bunnell is also appreciated.

3. Address: 1042 MHRI, The University of Michigan, Ann Arbor, Michigan 48104.

(Accepted for publication January 13, 1970) 